\title{
Comment revoir les filières AVC après l'introduction de la thrombectomie à la phase aiguë ?
}

\author{
How to Revise Stroke Pathways after the Introduction of Mechanical Thrombectomy?
}

\author{
M. Girot \\ Reçu le 16 février 2016; accepté le 14 mars 2016 \\ (C) SRLF et Lavoisier SAS 2016
}

Depuis la mise en place des unités neurovasculaires (UNV) et le déploiement de la thrombolyse IV, sa première étape structurelle, la filière AVC, est en constante évolution. Mais c'est une révolution qu'a déclenché la publication récente de cinq essais positifs (études MR CLEAN, ESCAPE, EXTEND-IA, SWIFT, REVASCAT) en imposant la thrombectomie mécanique (TM) dans le traitement de l'infarctus cérébral (IC) [1]. Il ne pouvait en être autrement compte tenu des résultats : $45 \%$ des patients traités par thrombolyse IV et TM complémentaire sont indépendants à trois mois vs $32 \%$ avec le traitement médical seul et ce, sans surrisque hémorragique [2]. Les sociétés savantes ont donc actualisé leurs recommandations : la TM est préconisée en complément de la thrombolyse IV dans le traitement de l'IC chez les patients présentant une occlusion proximale des artères de la circulation antérieure (carotide interne, cérébrale moyenne) dans les six heures après le début des signes ou en cas de contreindication à la thrombolyse IV [3]. Ce traitement pourrait concerner $15 \%$ des patients pour peu qu'ils soient rapidement identifiés.

Nous sommes au tout début de la procédure d'évaluation et pourtant déjà confrontés à un défi organisationnel pour que les résultats positifs des essais randomisés, menés dans des centres d'excellence, se concrétisent dans la « vraie vie $»$.

Le déploiement de la TM va se faire grâce au réseau des 133 UNV (sur les 140 prévues par le plan AVC 2010-2014) qui aujourd'hui assurent un maillage territorial quasiment complet et rendent possible la thrombolyse IV pratiquement sur tout le territoire. Même si des disparités existent, les UNV se préparent à cette évolution dans bon nombre de régions en se mettant en réseau avec les services d'urgence

M. Girot $(\bowtie)$

Service des urgences adultes, hôpital Roger Salengro, CHRU

Lille, rue Émile laine, F-59037 Lille

e-mail : marie.girot@chru-lille.fr

EA 2694 Épidémiologie et qualité des soins, Lille Université grâce à la télémédecine, et en renforçant leurs liens avec les UNV de recours comportant des services de neuroradiologie interventionnelle (NRI) et de neurochirurgie.

Les propositions d'optimisation de l'organisation vont reposer sur : (i) la sélection des patients; (ii) les délais de prise en charge ; et enfin (iii) la gestion des flux de patients à l'échelle des territoires de santé.

Pour mieux identifier les patients éligibles à la TM, il faudra, par des campagnes répétées, encore et toujours sensibiliser la population aux signes d'AVC, à l'existence de la filière de soins et aux possibilités thérapeutiques, alors même qu'aujourd'hui un patient sur deux ne fait pas appel au SAMU. À la question cruciale de savoir s'il est envisageable de sélectionner dès le préhospitalier les candidats à la $\mathrm{TM}$, la réponse est à ce jour négative : d'une part, la sélection repose sur un prérequis que constitue l'imagerie vasculaire non invasive (angioscanner ou angiographie par résonnance magnétique); d'autre part, il n'est pas envisageable de faire perdre du temps aux patients les plus nombreux qui peuvent bénéficier de la thrombolyse IV seule. Mais il existe des pistes de travail. Des expériences de thrombolyse préhospitalière reposant sur des systèmes d'imagerie embarquée ont été positives mais coûteuses [4]. La sélection en préhospitalier des patients les plus graves sur la base de score clinique vers un accès direct au centre de NRI est une stratégie possible mais dont l'efficacité n'est pas prouvée à ce jour à l'échelle d'un territoire de santé. En revanche, en intrahospitalier, si l'imagerie des vaisseaux n'est pas possible en routine, un score NIHSS $\geq 9$ dans les trois heures et $\geq 7$ dans les 6 heures peut être en faveur d'une occlusion des gros vaisseaux d'après les recommandations européennes [3]. Le déploiement de la télémédecine permettra par ailleurs de faciliter un accès rapide des patients à une imagerie pertinente.

Le deuxième enjeu de taille sera la gestion des délais. C'est le leitmotiv : plus que jamais la filière endovasculaire sera dépendante de l'efficacité de la filière thrombolyse. L'analyse des registres de thrombolyse IV a montré que nous 
étions loin de l'objectif préconisé de door-to needle time (DTNT) (délai admission-injection rt-PA) de moins de 60 minutes [5]. Les dernières recommandations soulignent à quel point il est important de monitorer les indicateurs d'efficacité de la filière [3]. Certains équipes préconisent de raccourcir la cible du DTNT à 30 minutes en ayant recours au scanner cérébral chez les patients pour lequel il n'y a pas de doute sur la clinique et le début des signes [6]. Le délai intrahospitalier recommandé entre l'admission et le point de ponction pour une procédure endovasculaire est de 120 minutes [7] ; pour certaines équipes, il devrait être en deçà, avec notamment une activation de l'équipe de NRI au moment de l'évaluation clinique [8]. Ces délais sont conditionnés par le type de sédation proposée, mais aussi et surtout par l'expérience du centre de recours. La marge de manœuvre sur les délais préhospitaliers reste plus étroite en l'absence de moyens supplémentaires. Ils n'ont d'ailleurs que peu évolué depuis quelques années en ce qui concerne la thrombolyse IV. L'évaluation de ces délais en soins primaires ou en transfert secondaire pour TM est nécessaire pour adapter les moyens de transports et l'orientation des patients. La filière est une succession de délais qui, pris globalement, peuvent impacter directement le pronostic du patient $[9,10]$.

Le dernier point est la gestion des flux de patients à l'échelle d'un territoire. La TM doit être réalisée par une équipe multidisciplinaire dans un établissement disposant d'une unité de soins intensifs neurovasculaires et d'un centre de NRI (38 centres autorisés en France). Compte tenu de l'activité croissante de TM attendue et de la pénurie en neuroradiologues interventionnels, l'option prise a été de renforcer les équipes existantes expérimentées plutôt que de faire appel à d'autres spécialistes comme les cardiologues interventionnels. Une montée en charge en termes d'effectifs devrait se faire à moyen terme sous l'impulsion de la Société française de neuroradiologie. L'évaluation capacitaire de l'UNV de recours en fonction de ses délais de retour vers l'UNV de proximité est une question d'actualité, l'objectif étant de ne pas pénaliser les soins de proximité assurés aux patients non thrombolysés.

L'évaluation des filières AVC par des indicateurs de qualité et d'efficacité, la mise en place de registres régionaux de thrombolyse IV et de TM sont des étapes nécessaires à la structuration, voire à la modification de la filière. L'arrivée de la TM en tant que traitement de référence va certes rendre plus complexe les filières de soins déjà organisées autour de la thrombolyse IV mais elle aura probablement un puissant effet structurant. Il aura fallu près de 15 ans à la thrombolyse IV pour devenir un traitement accessible à tous. L'enjeu des prochaines années est de garantir à tous, sur l'ensemble du territoire, l'accès au traitement endovasculaire.

Liens d'intérêts : L'auteur déclare ne pas avoir de lien d'intérêt.

\section{Références}

1. Mazighi M (2015) La thrombectomie : la deuxième révolution dans le traitement de l'infarctus cérébral. Réanimation 24:469-70

2. Badhiwala JH, Nassiri F, Alhazzani W, et al (2015) Endovascular Thrombectomy for Acute Ischemic Stroke: A Meta-analysis. JAMA 314:1832-43

3. Wahlgren N, Moreira T, Michel P, et al (2016) Mechanical thrombectomy in acute ischemic stroke: Consensus statement by ESOKarolinska Stroke Update 2014/2015, supported by ESO, ESMINT, ESNR and EAN. Int J Stroke 11:134-47

4. Lorenz MW, Lauer A, Foerch C (2015) Quantifying the Benefit of Prehospital Rapid Treatment in Acute Stroke: Benchmark for Future Innovative Clinical Trials. Stroke 46:3168-76

5. Fonarow GC, Zhao X, Smith EE, et al (2014) Door-to-needle times for tissue plasminogen activator administration and clinical outcomes in acute ischemic stroke before and after a quality improvement initiative. JAMA 311:1632-40

6. Kamal N, Benavente O, Boyle K, et al (2014) Good is not Good Enough: The Benchmark Stroke Door-to-Needle Time Should be 30 Minutes. Can J Neurol Sci 41:694-6

7. Qureshi AI, Abd-Allah F, Aleu A, et al (2014) Endovascular treatment for acute ischemic stroke patients: implications and interpretation of IMS III, MR RESCUE, and SYNTHESIS EXPANSION trials: A report from the Working Group of International Congress of Interventional Neurology. J Vasc Interv Neurol 7:56-75

8. Mehta BP, Leslie-Mazwi TM, Chandra RV, et al (2014) Reducing door-to-puncture times for intra-arterial stroke therapy: a pilot quality improvement project. J Am Heart Assoc 3:e000963

9. Sun CH, Nogueira RG, Glenn BA, et al (2013) "Picture to puncture": a novel time metric to enhance outcomes in patients transferred for endovascular reperfusion in acute ischemic stroke. Circulation 127:1139-48

10. Asif KS, Lazzaro MA, Zaidat O (2014) Identifying delays to mechanical thrombectomy for acute stroke: onset to door and door to clot times. J Neurointerv Surg 6:505-10 